\title{
Dominique Petit : parcours d'un danseur français aux États-Unis, rencontres et circulations - $\mathrm{N}^{\circ} 1$
}

Années 1970 et 1971 : des bords du Rhône vers New-York, premiers chocs chorégraphiques

Dominique Petit et Maëva Lamolière

\section{OpenEdition}

12 Journals

\section{Édition électronique}

URL : http://journals.openedition.org/danse/3622

DOI : $10.4000 /$ danse.3622

ISSN : 2275-2293

Éditeur

ACD - Association des Chercheurs en Danse

Référence électronique

Dominique Petit et Maëva Lamolière, «Dominique Petit : parcours d'un danseur français aux ÉtatsUnis, rencontres et circulations - No 1 », Recherches en danse [En ligne], Entretiens, mis en ligne le 12 février 2021, consulté le 25 février 2021. URL : http://journals.openedition.org/danse/3622 ; DOI : https://doi.org/10.4000/danse.3622

Ce document a été généré automatiquement le 25 février 2021.

association des Chercheurs en Danse 


\section{Dominique Petit : parcours d'un danseur français aux États-Unis, rencontres et circulations $-\mathrm{N}^{\circ} 1$}

Années 1970 et 1971 : des bords du Rhône vers New-York, premiers chocs chorégraphiques

Dominique Petit et Maëva Lamolière

\section{Introduction}

1 Le projet d'entretiens avec le danseur-chorégraphe Dominique Petit s'est fait de manière spontanée, sans cadre de recherche préalable ni de commande. Il est né de ma curiosité d'ancienne élève de Dominique Petit au regard de son parcours, dont il nous avait peu parlé pendant ses années d'enseignement. Cette nécessité s'est aussi accentuée quand j'ai commencé à me poser la question de la transmission de la danse. J'ai, à ce moment-là, ressenti le besoin de connaître davantage ce qu'il avait traversé pour, d'une certaine façon, nommer mes héritages, aussi indirects soient-ils. Dans un même mouvement, Dominique, de plus en plus conscient de la richesse de son parcours, avait lui aussi évoqué le désir de rendre visible son vécu de danseur, de chorégraphe et de pédagogue, pour « faire émerger des influences, des connections et des filiations qui risqueraient de rester dans l'ombre ou dans l'oubli ${ }^{1}$. "

2 C'est ainsi que cette aventure a commencé.

$\mathrm{Au}$ tout début, je pensais naïvement qu'une rencontre suffirait. Cependant, au bout de la première journée, c'est-à-dire après trois heures trente de discussion, Dominique n'avait retracé que les six ou huit premières années de son parcours, de ses débuts aux États-Unis jusqu'au milieu de l'aventure du GRTOP'. D'autres rencontres s'imposaient donc. Cette nécessité de poursuivre a été confortée car toutes les époques traversées, tous les contextes évoqués ont été aussi passionnants les uns que les autres. L'un comme l'autre nous nous sommes pris au jeu, au jeu de la parole, de la mémoire et de l'écoute. Du partage aussi - d'une autre forme de transmission. 
3 Je n'avais pas imaginé que la parole de Dominique serait aussi précise et passionnante et que son parcours avait été aussi foisonnant de rencontres et d'expériences. J'ai très vite eu la sensation que cette parole était pertinente car elle venait nommer une histoire de la danse ignorée, complexe, et que des enjeux forts en découlaient.

4 L'enjeu principal de ce processus d'entretien est d'ordre historiographique : il s'agit de nommer une histoire de la danse méconnue, voire insoupçonnée et d'en révéler les dynamiques et les circulations. Tisser une histoire qui rend compte de rencontres, de parcours, de lieux, de pratiques ; une histoire qui croise parfois les grands moments de la danse contemporaine mais qui lui apporte aussi distance, profondeur, complexité. Cette parole fait exister des creux, nuance les reliefs et permet d'autres prismes. Ce qui fait selon moi la pertinence de ce parcours, c'est qu'il va au-delà de la dimension autobiographique. En effet nous ne sommes pas - du moins je le crois - dans une égohistoire. La parole de Dominique se porte témoin d'une époque et convoque une communauté artistique, communauté bien souvent minoritaire que Mélanie Papin nommerait sans doute la communauté " des forces discrètes ${ }^{3}$ ». Par son témoignage, Dominique Petit nous révèle des expériences, des pratiques, des lieux, des rencontres et des moments féconds de l'histoire de la danse, entre les États-Unis et la France. Son parcours de danseur, de chorégraphe puis de pédagogue nous donne à penser ce qu'être artiste chorégraphique signifiait dans les années 1970-1980, avant l'explosion économique et institutionnelle de la danse. C'est donc aussi un témoignage sur les conditions économiques et les politiques culturelles de cette époque. Par ces multiples enjeux, ce parcours de vie s'adresse à des historien'ne's et des chercheurs'se's mais aussi plus largement à la communauté artistique parce que des liens de filiations, des forces au travail fécondes, souterraines et souvent impensées trouvent enfin une place dans ce que l'on appelle généralement « l'histoire de la danse ».

5 Nous faisons ici le pari du micro, du vécu intime, d'une histoire romanesque et biographique pour rendre compte d'une histoire bien plus large. Cette parole est certes parcellaire et a aussi ses omissions mais elle permet une plongée, une spécificité que les ouvrages généralistes ne nomment pas.

Dans nos échanges, Dominique se levait parfois et esquissait un geste. Il est aussi arrivé que nous regardions une vidéo ou des images pendant les entretiens que nous avons menés chez lui. Cependant, le passage à l'écrit ne permet pas de rendre compte de ces moments.

Nous nous sommes entretenus entre septembre 2015 et août 2016 dans des cadres conviviaux et amicaux, chez lui, chez moi ou encore au café. Douze heures d'entretiens ont été enregistrées. À ce temps de la parole, a suivi le temps de la retranscription, de la mémoire, des allers-retours dans ses archives personnelles pour vérifier les dates, les noms, essayer d'être au plus proche de ce qui a été, dans un souci historiographique toujours présent et auquel nous tenons.

La première série de ces entretiens concerne les quatre années que Dominique Petit a passé à New-York et qui marquent sa rencontre avec la danse. Nous avons découpé cette série en trois parties. La première ci-dessous concerne les années 1970 et 1971.

6 Afin de faciliter la lecture de ce récit, nous avons décidé de ne pas faire apparaitre les questions qui ont jalonné cette série d'entretiens. 


\section{Été 1970}

7 À l'origine de mon parcours en danse, c'est un stage de théâtre qui a tout déclenché.

Je venais de passer mon bac et je ne savais pas du tout ce que j'allais faire comme études. J'avais fait un bac philo et j'aurai bien aimé faire médecine, mais vu mon enthousiasme limité pour les études, ça me semblait un peu utopique, et pourtant, c'était la seule chose qui me paraissait envisageable dans la vie. Je faisais tout de même partie d'un petit cours de théâtre mis en place par la ville de Vienne en Isère où j'habitais alors. Cet été là, en 1970, juste après avoir passé mon bac, un stage de théâtre était proposé et je me suis inscrit. Ce stage était organisé par Catherine Dasté, la fille de Jean Dasté, qui était un des grands personnages de la décentralisation culturelle d'après la guerre. Il avait lieu à Châtillon sur Chalaronne, une petite cité médiévale dans les Dombes, au Nord de Lyon. Le stage devait durer deux semaines, et réunissait des professeurs de mime ${ }^{4}$, de chant, de danse, de cirque et de clown. Déjà en 1970, il y avait un esprit extrêmement ouvert sur le mélange des arts.

8 C'est dans ce cadre que j'ai pris mes premiers cours de danse, donné par Kilina Cremona ${ }^{5}$. Ces premiers cours ont été pour moi une révélation. Je n'avais jamais vu de spectacle de danse auparavant, j'avais seulement entendu parler de Maurice Béjart. Ma vision de la danse se résumait à deux choses : j'avais vu des photos du ballet du $\mathrm{XX}^{\mathrm{e}}$ siècle avec ces danseurs magnifiques, Jorge Donn, Paolo Bartoluzzi et Michaël Denard. Même en photo, on sentait qu'une image nouvelle de la danse apparaissait. L'autre représentation de la danse que j'avais venait d'un copain qui m'avait montré une photo de sa copine en tutu dans la forêt. En dehors de ces iconographies, je n'avais jamais vu un spectacle de danse car il n'y en avait pas à la télé, en dehors de la variété. J'imagine que j'avais dû voir des images de danseurs ou de danseuses classiques mais je n'avais jamais vu un danseur en mouvement. Kilina Cremona habitait à New-York et revenait passer l'été en France. Je pense qu'elle était la première française à être partie aussi longtemps étudier intensément avec Merce Cunningham. Je me souviens que ses cours étaient plus orientés vers la technique Graham que vers la technique Cunningham, mais cela était pertinent étant donné que nous étions tous très débutants. Finalement, peu importe la technique qu'elle nous a transmise car ces quelques cours ont été une découverte incroyable, découverte qui m'a suffi pour dire: "Je veux devenir danseur!». Cet élan était aussi entrainé par la personnalité de Kilina qui avait une gouaille et un enthousiasme communicatif. Il y avait là des forces avec lesquelles je n'étais jamais entré en contact.

9 Je faisais pas mal de sport à l'époque, beaucoup d'athlétisme, et surtout de la course de demi-fond. J'allais souvent courir en forêt ou au bord du Rhône pendant les cours de gym et, ce qui m'intéressait, au-delà de la compétition, c'était le fait de courir, en soi. En effet, j'avais traversé des états par la respiration qui étaient méditatifs. Je ne l'avais pas vraiment identifié comme tel, mais j'avais quand-même conscience qu'il se passait quelque chose de spécifique dans la répétition, dans le souffle et la transformation du souffle. J'avais surtout fait l'expérience que par le corps, on pouvait changer d'état. Après seulement quelques cours de danse avec Kilina, j'ai eu une sorte de vision que je résumerais ainsi : «dans la course à pied, le but c'est finalement la compétition et la trajectoire est rectiligne, alors que la danse m'apparaissait comme une étoile qui rayonne dans toutes les directions et dont la fonction serait la communication. » 

sur la vitre arrière de sa petite Volkswagen. C'était cette célèbre photo de Merce où on le voit pris dans un saut avec le bras ouvert sur le côté et le regard dans la même direction. Il est en l'air et, en même temps, il semble immobile, absolument calme et tranquille. Quand j'ai vu cette photo ça m'a totalement parlé, ça me semblait beaucoup plus fort que les images de Béjart.

11 En parallèle à cette découverte, il y avait également un projet familial qui consistait à m'envoyer passer un an dans une famille américaine pour prendre le temps de réfléchir à ce que je voulais faire. J'ai parlé de ce projet à Kilina [Cremona] qui a conforté cette initiative en me disant que New-York était vraiment le centre de la danse moderne et que je devais absolument m'y rendre. Deux ou trois mois plus tard, je débarquais à Washington, avec quelques sous gagnés en faisant les vendanges dans le Beaujolais. Ma famille d'accueil était la famille Mullany. Je tiens à la nommer parce qu'ils ont eux aussi été déterminants dans mes débuts en danse. Tous les frères et sœurs avaient les mêmes âges que les miens, ils étaient cinq et nous six. Je me suis retrouvé comme dans une deuxième famille et ils m'ont tout de suite adopté comme un des leurs. Chez nous, étant très proches en âge, on jouait beaucoup ensemble, mais chez les Mullany, on écoutait de la musique, on se mettait à chanter, on dessinait, le père écrivait des poèmes. C'était sans prétention, la vie s'exprimait comme ça chez eux. On riait beaucoup, c'était extrêmement joyeux. Grâce à cette éducation, le fils ainé, Steve Mullany, est devenu professeur de musique, l'une des filles, Joan Ballard tient maintenant une galerie d'art et le plus jeune, Thomas Mullany, est devenu un artiste renommé aux États-Unis. Rosemary, la mère de famille, n'avait pas de profession mais elle militait beaucoup dans les milieux sociaux et avait un petit peu de temps à me consacrer. C'est elle qui m'a aidé à trouver des cours de danse. Elle ne connaissait rien à la danse mais elle a pris les devants et cherché une école où je pourrais m'inscrire. Après quelques recherches, je me suis rendu à mon premier cours de danse classique chez une française déjà assez âgée qui avait une petite académie de danse dans le quartier de Georgetown. Le studio était sombre, tout en longueur et son mari pianiste accompagnait le cours. La leçon devait avoir lieu vers dix-huit heures, et je me suis retrouvé au milieu de petites filles de sept ou huit ans habillées en justaucorps rose. Ce n'était pas tout à fait ce que j'avais connu avec Kilina [Crémona], mais je découvrais les rudiments de la danse classique et du travail à la barre.

En très peu de temps, quelques évènements m'ont profondément marqué et m'ont totalement convaincu de poursuivre dans cette nouvelle voie. Le premier de ces évènements a été d'assister à mon premier spectacle de danse. Je n'avais jamais vu de danse moderne et je ne savais pas du tout à quoi m'attendre. C'était Rumble de Dan Wagoner, ${ }^{6}$ un duo avec son compagnon de vie. Son compagnon, poète et vivant aussi de menuiserie, était là avec ses outils et travaillait le bois tout en disant des poèmes pendant que Dan Wagoner dansait autour. Il avait une danse très vive, très enjouée, on aurait dit une sorte de lutin. En même temps, le mouvement semblait très naturel, comme improvisé. Je ne crois pas qu'il y avait de musique, tout le son était constitué des bruits de travail sur le bois : scier, raboter, visser, clouer et les poèmes dit à haute voix, par intermittence. Cela créait une ambiance calme, un peu méditative, qui faisait 
ressortir la vivacité de la danse. J'étais évidemment très surpris, mais aussi, pour la première fois, très touché par un spectacle. Après cela, je me sentais complètement en décalage avec les quelques cours de classique que je prenais.

Le deuxième évènement n'est pas un spectacle mais un stage. Une autre professeure, que j'avais rencontrée m'a fait part d'un stage où Paul Sanasardo ${ }^{7}$ allait enseigner. J'ai suivi son conseil et je me suis retrouvé dans un grand gymnase avec une estrade au milieu qui pouvait contenir cent-cinquante personnes. Je n'avais jamais vu ça. Ce fameux professeur était un homme à la carrure imposante qui donnait le cours en s'accompagnant au tambour et en chantant avec une voix très puissante. Je me souviens d'un personnage extrêmement charismatique, on aurait dit une sorte d'indien tellement il paraissait habité, sa manière de chanter, de psalmodier pendant le cours. Je commençais tout juste la danse, c'était un vocabulaire que je découvrais totalement mais cette rencontre a de nouveau suscité chez moi un désir de danse. Les cours de Paul Sanasardo se composaient d'exercices assez simples qui me permettaient de me débrouiller malgré mon faible bagage technique. Il y avait des choses que je ne pouvais pas saisir sur l'instant, mais je crois que ce n'était pas trop catastrophique. À la fin du stage, la professeure qui m'avait amené là m'a présenté à Paul. Très chaleureux, il m’a donné l'adresse de son studio à New-York en soulignant que des danseurs français de l'Opéra y étaient passé peu de temps avant. Après ce stage, je suis allé voir le spectacle de sa compagnie. Comme la plupart des spectacles de danse de l'époque, ce programme était composé de trois ballets. Je me souviens d'espaces esthétiques inouïs pour moi et tout me paraissait extrêmement original.

Quelques temps après, Mme Mullany a pris contact avec le département danse de l'université de Washington pour se renseigner sur les formations en danse. Par chance, un stage ouvert à tou'te's allait avoir lieu. C'est Yvonne Rainer ${ }^{8}$ qui donnait le stage et transmettait Chair and Pillow dance. On était une vingtaine d'étudiant.es et nous avons tous appris Chair and Pillow dance avec elle! Elle était très simple, joyeuse et décontractée. Le stage s'est déroulé dans la bonne humeur, sans aucun stress car elle savait nous mettre en confiance et nous avait même dit que, si on se trompait, ce n'était pas grave. Chair and Pillow dance a été donné en fin de stage à l'auditorium de l'Université et c'était la première fois que je dansais sur scène, le jour de mes vingt ans ! Un autre événement extraordinaire eu lieu juste après: Yvonne Rainer était à Washington avec le Grand Union pour donner une performance au Smithsonian Museum?. Je ne sais plus comment ils avaient intitulé cet événement, probablement pas un «event » car c'était plutôt le mot qu'utilisait Merce Cunningham. Il s'agissait d'une performance essentiellement basée sur de l'improvisation. Le Smithsonian Museum c'est un très grand musée, un peu comme le musée Orsay, juste côté du Capitole. Cet évènement eut lieu au moment des manifestions pour l'arrêt de la guerre au Vietnam et j'ai appris plus tard par Yvonne Rainer, que, jusqu'au dernier moment, elle ne savait pas si le département d'État allait autoriser cette représentation en raison de toute l'agitation politique ambiante. En effet, on était en plein dans cette tension de la guerre du Vietnam et, évidemment, le positionnement du Grand Union était extrêmement contestataire. C'était une performance sous forme de parcours déambulatoire et les danseur'se's occupaient tout l'espace : les escaliers, les grands couloirs, les salles et le public circulait comme il voulait. Á un moment donné, je me suis retrouvé à regarder plusieurs danseurs qui évoluaient et Yvonne Rainer s'est rapprochée de moi avec cette faculté de rester toujours très ouverte face au public. Elle m'a reconnu et m'a fait signe de venir danser avec eux! Comme je regardais depuis un moment, j'avais fini par 
décoder les jeux et les codes utilisant des mouvements quotidiens, tout en jouant avec le temps et avec l'espace environnant. Sans aucune hésitation, j'ai enlevé mon blouson et je suis allé improviser avec eux elles les vingt dernières minutes de la performance!

Trente-cinq années plus tard, j'ai croisé Yvonne Rainer à Nantes et je tenais absolument à lui reparler de cet évènement. Je lui ai raconté ce qui s'était passé et elle m'a répondu qu'elle ne s'en souvenait pas, mais que c'était une histoire magnifique.

Voilà comment je suis entré dans la danse. J’ai vécu tout cela comme un rêve éveillé, dans un état d'étonnement constant. Je poursuivais tout de même ces petits cours de ballet mais j'avais aussi été prendre des cours plus sérieux. Par chance, cette professeure française avait des relations dans le monde de la danse classique et m'avait obtenu une bourse pour étudier à l'école du National Ballet! On m'avait dit que, pour commencer, je devais prendre les cours pour garçon. Nous n'étions que trois ou quatre dans ce cours et les autres danseurs étaient beaucoup plus avancés que moi. J'ai aussi pris des cours de partnering, mais j'avais la sensation étrange que tout cela sentait un peu la poussière et ces ambiances de travail ne m'attiraient pas. On me disait que pour être danseur, il fallait faire de la danse classique mais cela ne me plaisait pas. En pleine répétition de Casse Noisette avec le National Ballet, Kilina [Cremona] m'a téléphoné pour me dire que Merce [Cunningham] donnait un stage d'une semaine pendant les vacances de Noël. Sans hésiter, je me suis inscrit et je suis parti une semaine à New-York !

Je campais sur un matelas chez Kilina [Cremona] qui avait bien voulu m'accueillir. Elle habitait sur la deuxième avenue, quasiment à l'angle de la 8ème, c'est-à-dire au cœur $\mathrm{du}$ quartier hippie de cette époque. Une boutique sur deux était un magasin d'accessoires pour fumer de la marijuana ou bien de manteaux afghans en peau de chèvre car c'était la grande mode. On aurait dit que tous les danseurs habitaient plus ou moins le quartier. En tous cas, le studio de Merce [Cunningham] n'était pas très loin et se situait un peu plus haut sur la $2^{\mathrm{e}}$ avenue. Les cours étaient ouverts à des profils très différents et on ne m'avait pas du tout demandé mon niveau pour m'inscrire. Je me souviens être à côté de danseur'se's très avancée's, notamment Myriam Berns ${ }^{10}$ qui m'impressionnait tellement, avec ses jambes immenses et un coup de pied comme je n'en n'avais jamais vu! Il devait y avoir quarante ou cinquante personnes à chaque cours, mais j'avais l'impression que nous étions cent-cinquante! J'ai pataugé complètement car le niveau était trop élevé pour moi qui n'avais pris qu'une semaine de cours sérieux avec Paul Sanasardo. J'en suis sorti un peu frustré, mais fier de l'avoir fait ! Je garde la vision d'un studio délabré, les murs pelaient, le sol était assez ondulé par endroits mais l'ambiance, la motivation des danseurs qui étaient là et la façon dont ils prenaient le cours avaient quelque chose d'extraordinaire. J'imagine que Merce [Cunningham] ${ }^{11}$ donnait le cours, mais je ne me souviens plus. Je n'avais jamais vu la compagnie Cunningham danser sur scène mais je traversais et voyais des sensations de mouvements extraordinaires parce que la danse me semblait être hors de toute représentation. Il s'agissait juste de faire. Je suis reparti de cette semaine de stage avec le désir urgent de revenir à New-York.

\section{Année 1971}

Rentré à Washington, je me suis mis à faire des petits boulots de restauration et j'ai débarqué à New-York avec soixante-dix dollars en poche. J'ai prévenu la famille Mullany de mon départ et j'ai appelé Kilina [Cremona] qui a accepté de m'accueillir. Je 
pense qu'elle souhaitait rentrer dans la compagnie de Merce Cunningham mais en attendant, elle suivait les cours professionnels avec la compagnie et donnait des cours au studio. Je sais qu'à un moment elle a donné les cours pour «intermédiaires ». En parallèle à l'enseignement, elle travaillait pour Merce Cunningham comme cuisinière. En effet, quand il avait besoin de réunir tous ceux qui lui donnaient des fonds, il organisait un grand repas pour eux, dans le studio. Cette activité parallèle à l'activité artistique témoigne aussi des modes de vie des danseurs et danseuses de cette époque. Nous vivions tous et toutes avec des petits boulots alimentaires annexes. Après le souvenir de mon premier stage avec Merce Cunningham et celui de Paul Sanasardo, il m'a semblé qu'il valait peut-être mieux me diriger vers les cours de Paul, et je suis donc allé frapper à son studio. Il m'a accueilli en m'invitant à suivre le cours débutant/ intermédiaire de $17 \mathrm{~h} 30$. Le cours de la compagnie ouvert aux professionnels avait lieu après, à $19 \mathrm{~h}$. En règle générale, les studios étaient réservés dans la journée pour les répétitions et le soir pour les gens qui venaient pratiquer, d'abord les amateurs, puis les professionnels. J'ai donc commencé à prendre les cours de $17 \mathrm{~h} 30$ que donnait Paul [Sanasardo]. C'est lui qui donnait les deux cours à la suite, après sa journée de création avec la compagnie. C'était des exercices extrêmement simples, très décomposés, auxquels j'avais accès tout de suite parce qu'il n'y avait pas de coordinations trop complexes. La structure du cours, de chaque exercice même, sa rythmique, tout était basique et assez "classique » : on commençait à la barre, une barre très académique mais très simple pendant une demi-heure. Puis s'enchaînaient une série d'exercices au sol, très «Graham " puis un "milieu » tel qu'on le retrouve dans les cours de danse classique, avec un adage, des sauts et puis de grandes traversées, toujours très dynamiques. Paul improvisait les adages, les traversées sur le moment mais c'était toujours extrêmement structuré. Les cours étaient accompagnés par un musicien, donc même en étant extrêmement simplifiée, la relation à la musique était présente dans l'apprentissage et la structuration du cours. Cela m'arrangeait parce que je ne connaissais rien en musique, je ne savais pas ce qu'était une portée, ni même une mesure, une blanche ou bien une noire. Techniquement, cette combinaison de classique et de Graham était extrêmement efficace pour la construction des corps. Les traversées, elles, étaient plutôt modernes alors que l'adage était presque néo-classique. Paul avait commencé la danse vers dix-neuf ans en prenant des cours chez Martha Graham. Assez vite, il a eu envie de faire du classique et il a étudié avec Mia Slavenska ${ }^{12}$. Il avait beaucoup travaillé avec elle et toute la barre était directement issue de l'enseignement de cette danseuse. Paul lui-même était devenu un très beau danseur grâce au mélange de ces deux techniques. C'est étonnant parce que, à partir d'éléments finalement assez basiques, cela construisait les corps en profondeur et comme les cours étaient très simples, j'ai pu me construire avec ça.

Dans ce studio j'ai fait la rencontre de Jacques Patarozzi ${ }^{13}$, danseur arrivé chez Paul avec sa compagne de l'époque, six mois ou un an avant moi. Il était issu du jazz, avait fait de nombreux shows pour la télévision et avait travaillé pour de nombreuses célébrités dont Johnny Halliday. D’origine Corse, il était venu à Paris pour faire de la variété et avait développé un réseau conséquent. Avec Michelle Rebaud, sa compagne également danseuse, ils ont décidé de partir à New-York pour découvrir de nouveaux univers artistiques. Je ne sais pas où ni comment il a rencontré Paul, mais il y est resté. Quand je suis arrivé, ils étaient là depuis un certain temps déjà et avaient un très bon niveau. Ils prenaient donc le cours "professionnel» de dix-neuf heures avec la compagnie. J'étais heureux d'échanger avec des Français·e·s et nous nous sommes tout 
de suite très bien entendu·es. Les autres Français·e's dont Paul m'avait parlé étaient repartiees depuis longtemps. Il s'agissait Jacques Garnier ${ }^{14}$ accompagné d'un petit groupe issu du Ballet Théâtre Contemporain, dont Quentin Rouiller ${ }^{15}$, avec qui je danserai plus tard au sein du GRTOP ${ }^{16}$. Ces danseur.se's étaient passéeess prendre les cours de Paul Sanasardo parce qu'il était connu à New-York comme un très grand professeur. À partir de ce moment-là, j'ai vécu de petits boulots et je prenais mon cours quotidien au studio de Paul Sanasardo. La famille Mullany m'avait également mis en contact avec une autre famille à New York, chez qui j'ai vécu "au pair », pendant plus d'un an. Ils habitaient les quartiers chics, près de la $5^{\text {ème }}$ avenue, je m'occupais un peu des deux enfants, je promenais le chien et je faisais la cuisine. Je gagnais 30 dollars par mois ce qui était exactement la somme mensuelle que Paul m'avait demandée, ce qui faisait un peu plus d'un dollar par cours, pour 6 cours hebdomadaires. Ce n'était pas cher mais je n'avais pas le moindre argent pour le reste. J'ai fait part de ma difficulté financière à Paul qui m'a proposé de nettoyer le studio de danse en échange des cours. Je passais donc toute ma journée du dimanche à nettoyer tout le studio. Je venais le matin, et j'avais le studio pour moi. J'étais assez heureux d'être là, dans cette ambiance et Paul trouvait le lundi matin le studio extrêmement propre!

D'autres rencontres absolument précieuses ont aussi jalonné cette période. En effet, j'avais également obtenu une bourse pour prendre des cours de classique au Jeoffrey Ballet School grâce à l'administrateur de la compagnie de Paul Sanasardo, William Weaver. Je pouvais prendre tous les cours gratuitement mais je n'étais pas assez assidu car je ne trouvais pas les cours très intéressants. C'était une bonne école de danse classique, mais à côté des cours de Paul [Sanasardo], j'avais du mal à y trouver du sens. Paul [Sanasardo] était extrêmement investi dans son enseignement; il nous corrigeait et pouvait interrompre le cours quinze minutes pour nous parler de musique, de peinture ou de littérature. Je me souviens qu'il parlait souvent de Jean Genet et d'André Gide. Officiellement, j'étais étudiant et boursier de la Joffrey Ballet School : cela avait le double avantage de me permettre d'avoir un visa étudiant et de pouvoir obtenir plusieurs sursis consécutifs pour mon service militaire. Cela a été en partie possible grâce au mari de Françoise Adret ${ }^{17}$ qui travaillait à l'ambassade de France (ou au consulat) et qui connaissait très bien la danse et les problèmes des danseur'se's. Chaque année, par l'intermédiaire de l'ambassade de France, je pouvais justifier que j'étais vraiment étudiant grâce à cette bourse.

J'ai dû arriver chez Paul Sanasardo à la mi-février 1971 et, au cours de l'été suivant, il y avait un stage d'été de la compagnie comme cela se faisait beaucoup à l'époque. Le stage avait lieu dans un centre d'art à Saratoga Springs. Saratoga Springs est une ville située au nord de New-York, en direction du Québec. C'est un peu le « Vichy » des ÉtatsUnis. En effet, c'est également une ville d'eau, qui a été aussi le lieu d'une grande bataille. Et puis, c'est un lieu de villégiature comme Vichy, avec en plus des établissements de thermes, un champ de course et une salle de concert immense qui chaque été accueillait en résidence le célèbre Philadelphia Symphonic Orchestra. C'est un peu comme si l'opéra de Vichy avait en résidence l'orchestre de Berlin ; il n'y avait que des très grands chefs d'orchestre. Je crois que le New-York City Ballet venait en juillet et puis juste après, en août, la compagnie de Paul Sanasardo donnait un stage et une ou deux représentations. Paul [Sanasardo] m'avait fortement conseillé de venir 
prendre le stage et il me semble que je n'avais rien à payer. Évidemment, ce stage attirait beaucoup de danseur.se's, dont certaine's qui voulaient travailler avec Paul [Sanasardo]. Ce dernier donnait lui-même des cours mais certain'e's des danseur-se's de la compagnie prenaient aussi le relai : Diane Germaine, Jerry Houlihan et Manuel Alum, son danseur principal qui était aussi un remarquable chorégraphe. En fin de stage avait lieu une présentation des chorégraphies écrites pour les étudiant·es en première partie du spectacle de la compagnie. Le soir, toute's les stagiaires avaient des entrées pour aller écouter des concerts en plein air donnés par le Philadelphia Symphonic Orchestra. Je me souviens avoir écouté, allongé sur l'herbe, la 5eme symphonie de Mahler, dirigée par Bruno Walter !

Cette année-là, Paul [Sanasardo] avait invité Pina Bausch ${ }^{18}$ à venir enseigner le temps du stage. Ils se connaissaient parce que Pina [Bausch] avait déjà dansé pour lui. En effet, elle avait passé deux ans à New-York, entre 1958 et 1960, et rencontré pleins de gens, dont Antony Tudor, ${ }^{19}$ un grand chorégraphe américain dont Paul [Sanasardo] était très proche. Paul [Sanasardo] et Antony [Tudor] étaient très amis et entretenaient une relation artistique intense et régulière. Quand Antony Tudor et Paul [Sanasardo] ont vu Pina [Bausch] danser, ils l'ont immédiatement contactée. Elle dansa pour Paul Sanasardo mais aussi pour Paul Taylor et Antony Tudor. Ce concours de circonstances m'a donné la chance de prendre des cours avec Pina [Bausch]. Je crois qu'elle ne serait pas offensée si je dis qu'à cette époque, elle ne semblait pas vraiment aimer enseigner, on avait l'impression que ça ne l'intéressait pas du tout! Son cours était très particulier : on était à la barre, en silence, il n'y avait pas d'accompagnateur-trice. Elle montrait des longues séquences de mouvements qui lui appartenaient totalement, avec un phrasé très spécial, fait d'accents, de ruptures au milieu, d'un flux extrêmement fluide, absolument sans efforts! Nous avions tous'tes beaucoup de mal à suivre, et elle ne nous donnait presque aucune indication et ne nous corrigeait pas. C'est en la voyant danser plus tard que j'ai compris son style mais, pédagogiquement, ce n'était pas très efficace. Je n'ai pas du tout échangé avec elle cet été-là. Elle était une grande artiste et moi j'étais juste un français débutant ; nous n'avions pas grand-chose à partager.

Lors de ce stage, Paul [Sanasardo] a créé un ballet pour les étudiant·es et il m'avait mis en scène dans un ballet avec les autres stagiaires. De plus, Manuel Alum avait créé une pièce dans l'atelier où j'étais et que j'avais beaucoup aimé. C'était une sorte d'ambiance de cirque, avec tout le monde sur le plateau, dans une écriture très ouverte. Je me souviens par exemple d'avoir fait une roue au ralenti, au milieu des autres danseur'se's, très près d'euxeelles, en les frôlant presque. C'était une sorte de suggestion ou d'évocation du cirque. Des sensations de jonglage mais ce n'était pas jongler, des sensations d'équilibre, mais ce n'était pas descriptif et la composition était très délicate. J'aimais beaucoup cet espace un peu kaléidoscopique, non hiérarchisé et cette juxtaposition d'éléments disparates créait un tableau animé. J'avais vraiment l'impression que cette composition et cet espace scénique m'ouvraient des espaces intérieurs et je pensais que cela devait faire la même chose au public. Je me souviens que pendant ce stage, on prenait des cours du matin au soir, et que certains jours nous enchaînions onze heures de cours et de répétitions! C'était énorme mais j'adorais ça, j'étais gourmand de danse, ne loupais aucun cours et me souciais peu de mon état de fatigue. 
Après ce stage, l'idée de repartir en France était impensable et j'ai décidé de rester à New York. L'année d'après, Malou Airaudo ${ }^{20}$ est arrivée car elle avait rencontré Manuel Alum $^{21}$, alors invité à enseigner au Ballet Théâtre Contemporain à Amiens. Malou [Airaudo] venait du classique, de l'Opéra de Marseille qui était alors dirigé par Joseph Lazzini, et elle avait ensuite poursuivi au Ballet Théâtre Contemporain (BTC), dirigé à l'époque par Jean-Albert Cartier me semble-t-il. Le Ballet Théâtre Contemporain a été une aventure assez formidable. Pour résumer, le projet était de refaire quelque chose comme les Ballets Russes, en passant commande aux plus grand.es artistes du moment. Le BTC était implanté à la maison de la culture d'Amiens et faisait partie du mouvement de décentralisation culturelle initié dans les années 1960. Il s'agissait de créer en France un projet chorégraphique innovant, en invitant les plus grandeess chorégraphes, les plus grand·e's plasticien'ne's et les plus grand·e's musicien'ne's. Il y a eu des créations assez inouïes qui ont été faites. Le BTC se composait d'un groupe de quarante danseurs, dont Dominique Mercy, Quentin Rouiller, James Urbain, Norio Yoshida, des danseurs de formation classique donc. Malou Airaudo faisait partie de cette troupe et était peut-être même soliste. Le BTC avait invité Manuel Alum, pour donner des cours et c'est là qu'ils se sont rencontrés. J'imagine que le BTC avait voulu inviter Paul Sanasardo mais que, n'étant pas libre, il avait envoyé Manuel [Alum] car c'était un danseur tout à fait représentatif de cette technique-là. Il était d'origine portoricaine et avait débarqué à New York et Paul Sanasardo l'avait rapidement repéré. Il était devenu le danseur fétiche de sa compagnie. Il avait un corps extrêmement fin et délié avec une ligne stupéfiante. Il développait parallèlement ses propres chorégraphies et cherchait aussi à monter sa compagnie. Je crois qu'il souhaitait s'affranchir de la « tutelle » de Paul et il y avait pas mal de rivalités entre eux deux à ce moment-là parce que Manuel [Alum] avait justement besoin de voler de ses propres ailes. Ses pièces étaient très singulières, et dans un sens, moins académiques que celles de Paul [Sanasardo]. J'ai surtout vu ses soli, notamment Cellar qui était inouï, d'une puissance vraiment extraordinaire.

Paul [Sanasardo] m'a un jour annoncé l'arrivée de Malou Airaudo et je suis allé la chercher à l'aéroport. C'était une jeune femme un peu plus âgée que moi, mais comme je paraissais avoir dix-sept ans à cette époque, elle faisait beaucoup plus mûre. En arrivant à New York, elle avait l'air un peu perdue et je crois qu'elle ne parlait pas anglais, j'étais donc son " guide » et son interprète. À ce moment-là, elle traversait une forme de dépression et était arrivée à New-York pour prendre l'air et découvrir de nouveaux horizons. Cependant, elle a très vite déchanté car Manuel [Alum] n'avait pas de compagnie et pas de projets stables. Il faisait un spectacle par an et il ne pouvait payer personne pour les répétitions. Elle est restée et a attendu qu'il y ait une " saison » à New-York. A cette occasion, Manuel [Alum] lui a écrit un magnifique solo, Woman of mystic body et elle a également dansé une autre pièce, un magnifique trio de Manuel qui s'appelait Palomas, avec Jennifer Muller et Willa Kahn. Puis il y a eu Sextetrahedron, dont je n'ai presque plus de souvenirs. En attendant que les projets prennent forme, elle était ravie de découvrir New-York, de rencontrer Paul [Sanasardo], de s'imprégner de l'ambiance du studio et elle s'y est tout de suite plu. Elle est donc restée un certain temps. Elle prenait les cours de la compagnie ainsi que des cours de classique avec Maggie Black ${ }^{22}$ qu'elle aimait beaucoup. Et, de temps en temps, nous allions aussi prendre des cours avec Karel Shook ${ }^{23}$ qui enseignait à l'école du Dance Theater of Harlem. 
Il y a une expérience qui m'a particulièrement marqué, probablement cette année-là. J'étais allé me promener dans Central Park et, à un moment, j'ai aperçu un groupe de musiciens qui jouaient des steel drums, ces instruments fabriqués à partir de grands bidons métalliques. C'était la première fois que j'en voyais et que j'en entendais jouer. Les musiciens devaient être d'origine latino-américaine et leur musique avaient des couleurs très afro-cubaines, ou des caraïbes, je ne suis pas un spécialiste. J'ai rejoint le groupe des auditeurstrices qui les entouraient, et, très vite, j'ai senti que cette musique pénétrait tellement à l'intérieur de moi que, si je continuais à l'écouter, je ne pourrais pas m'empêcher de me mettre à danser. Je me sentais complètement attiré, mais j'avais en même temps le sentiment d'être dominé par la musique, comme si celle-ci avait le pouvoir de m'emmener dans des zones hors de contrôle. Quand j'ai réalisé ça, je me suis dit qu'il fallait mieux que je m'éloigne car je sentais que je n'étais pas prêt à vivre cela et je n'avais absolument pas envie de me donner en spectacle. Je suis resté assez longtemps troublé par cette expérience. Je sentais que, d'une certaine façon, elle me parlait profondément, dans ma relation à la danse. J'avais pour la première fois senti une certaine forme de pouvoir de la musique, mais surtout, j'avais touché un aspect de la danse qui peut côtoyer la transe, et même s'y confondre. J'ai senti qu'il y avait là une dimension qu'il me faudrait explorer un jour, pour ne pas rester «à quai » comme je l'avais fait ce jour-là.

L'année s'est passée comme ça, je prenais de plus en plus de cours au studio, quelquefois les deux cours à la suite, j'avais la sensation de progresser très rapidement, et je n'envisageais rien d'autre que cette vie-là. J'étais tellement bien à cet endroit, c'était tellement familial; avec Jacques [Patarozzi], Michelle [Rebaud] et Malou [Airaudo], on faisait une petite bande de français très soudée.

\section{NOTES}

1. Petit Dominique, entretiens personnels.

2. Groupe de Recherche Théâtrale de l'Opéra de Paris.

3. PAPIN Mélanie, 1968-1981: construction et identités du champ chorégraphique contemporain en France : désirs, tensions et contradictions, Thèse de doctorat en danse, sous la direction d'Isabelle Launay, département danse, université de Paris 8, 2017.

4. Dont Gérard Lebreton, qui avait travaillé avec Jean Louis Barrault et Marcel Marceau.

5. Kilina Cremona est une danseuse, chorégraphe et pédagogue française. Formée à Paris chez Karin Waehner et à New York au Studio Cunningham, elle danse aussi pour Meredith Monk, David Gordon, Twyla Tharp et Viola Farber. Kilina Cremona rentre en France en 1980 et crée sa propre compagnie. Après s'être installée à Zagreb en Croatie à partir de 1995, elle revient s'installer à Lyon en 2002 et ouvre les ateliers Desmaé qui entretiendront un partenariat avec le cursus « danse études » de l'université Lyon 2.

6. Dan Wagoner est un danseur et chorégraphe américain. Après avoir dansé chez Martha Graham, Merce Cunningham et Paul Taylor, il crée sa compagnie Dan Wagoner and dancers de 1969 à 1994. Il est aujourd'hui professeur au département danse de l'université de Floride. 
7. Paul Sanasardo est un danseur et chorégraphe américain d'origine italienne. Diplômé de l'école des arts de Chicago il a ensuite étudié la danse avec Anthony Tudor et Martha Graham. Il fonde en 1957 la compagnie Paul Sanasardo-Donya Feuer. Pour plus d'informations, je renvoie le lecteur à l'ouvrage de Mark Franko: FRANKO Mark, Excursion for miracles : Paul Sanasardo, Donya Feuer and studio for dance 1955-1964, Middletown, Wesleyan University Press, 2005.

8. Yvonne Rainer est une artiste chorégraphique américaine qui appartient au courant de la "post-modern dance ». Formée à la danse classique, chez Martha Graham et chez Merce Cunningham, elle rencontre dans les années 1960 Anna Halprin et participe à la création du Judson Church Dance Group en 1962. Dans les années 1970 elle s'intéresse aussi au cinéma et réalise des longs métrages expérimentaux.

9. Probablement War, pièce créée en 1970.

10. Myriam Berns est une danseuse et pédagogue américaine. Après une formation chez Merce Cunningham, elle s'implante en France et enseigne la technique Cunningham.

11. Merce Cunningham est un danseur et chorégraphe américain. Après avoir été danseur chez Martha Graham, il crée ses premiers soli dès 1945 avant de fonder sa compagnie en 1953 au Black Mountain College. Il collabore, entre autres, avec Robert Rauschenberg et John Cage et produit des formes chorégraphiques très diverses. Pour que ses danseur'se's puissent être disponibles à ses propositions chorégraphiques, il établit une série d'exercices (on parle alors de technique Cunningham) et met le principe du hasard au cœur de son processus chorégraphique.

12. Mia Slavenska est une ballerine américaine d'origine croate. Après une formation en Croatie, elle devient première danseuse des Ballets de Monte-Carlo. Elle fonde sa propre compagnie, Ballet Variante en 1944 à Hollywood et devient première danseuse du Metropolitan Opera de New-York en 1954. À partir des années 1960, elle se consacre à l'enseignement.

13. Jacques Patarozzi est un danseur d'origine Corse. Il intègre le Tanz Theater de Wuppertal de Pina Bausch en 1978. Après avoir créé sa propre compagnie, la compagnie Balmuz en 1982, Jacques Patarozzi initie en 1999 le festival Le printemps de la danse en Charente. Il dirige le Théâtre de l'avant-scène de Cognac de 2009 à 2014 et il est ordonné moine Zen en 2004.

14. Jacques Garnier est un danseur et chorégraphe français qui a dansé dans le corps de ballet de l'Opéra de Paris avant de partir se former aux États-Unis auprès de Alvin Nikolais et Merce Cunningham. Avec Brigitte Lefèvre, il crée en 1972 le Théâtre du Silence, compagnie implantée à La Rochelle. Il fonde en 1981 le GRCOP (Groupe de Recherche Chorégraphique de l'Opéra de Paris) et en assure la direction artistique jusqu'à sa mort en 1989.

15. Quentin Rouiller est un danseur, chorégraphe et pédagogue français. Formé à la danse classique, il danse au Ballet Théâtre Contemporain puis au Théâtre du silence. Il participe ensuite à l'aventure du GRTOP (Groupe de Recherche Théâtrale de l'Opéra de Paris) dirigé par Carolyn Carlson et crée sa propre compagnie, la compagnie « Moebius » en 1977. Il prend la direction des études chorégraphiques du Conservatoire National Supérieur de Musique et de Danse de Paris de 1989 à 1999.

16. Le GRTOP (Groupe de Recherche Théâtrale de l'Opéra de Paris) est créé en 1975 par Carolyn Carlson tout juste nommée "étoile» par Rolf Liebermann. Ce groupe de travail dirigé par Carolyn Carlson, dont Dominique Petit fera partie, développe de manière conséquente l'improvisation et crée une vingtaine de pièces chorégraphiques. Il prend fin en 1980.

17. Françoise Adret est une danseuse, chorégraphe et pédagogue française. Après une carrière de danseuse étoile et de maitresse de ballet, elle crée en 1968 le Ballet Théâtre Contemporain avec Jean-Albert Cartier à la maison de la culture d'Amiens. Le BTC s'implante ensuite à Angers en 1972. Françoise Adret est ensuite inspectrice de la danse de 1978 à 1985 et dirigera aussi de nombreux ballet en France et à l'étranger.

18. Pina Bausch est une danseuse et chorégraphe allemande. Formée à la Folkwang-Hochschule d'Essen par Kurt Joss et Jean Cébron, elle complète sa formation aux États-Unis à la Juilliard School à New-York en 1958. Elle rencontre aux États-Unis Paul Taylor, Antony Tudor et Paul 
Sanasardo entre autres. En 1973, elle est nommée à la tête du Tanztheater de Wuppertal. Elle crée dans un premier temps des chorégraphies sur des opéras avant de réinventer ses méthodes de travail à la fin des années 1970 et développer des formes chorégraphiques qualifiées de «danse théâtre ".

19. Antony Tudor est un danseur et chorégraphe britannique. Il débute sa carrière à Londres au sein du ballet Rambert avant d'intégrer l'American Ballet Theater comme danseur et chorégraphe invité.

20. Malou Airaudo est une danseuse française formée à la danse classique. Après une carrière dans différents ballets, elle intègre en 1974 le Tanz Theater de Wuppertal, dirigé par Pina Bausch, dont elle devient une des interprètes principales.

21. Manuel Alum est un danseur et chorégraphe américain d'origine portoricaine. Il étudie auprès de Martha Graham, de Mia Slavenska et de Karel Shook avant de rejoindre la compagnie de Paul Sanasardo en 1962. En 1972 il crée sa compagnie, la Manuel Alum Dance Company.

22. Maggie Black est une danseuse et pédagogue américaine qui a développé une technique de danse classique fondée sur l'anatomie. De nombeux'ses danseur'se's classiques et modernes ont suivi ses cours.

23. Karel Shook est un maitre de ballet, chorégraphe et écrivain américain. Avec Arthur Mitchell, il co-fonde en 1968 le Dance Theater of Harlem.

\section{RÉSUMÉS}

Ce premier volet d'entretiens, "Années 1970 et 1971: des bords du Rhône vers New-York, premiers chocs chorégraphiques ", revient sur la rencontre entre Dominique Petit et la danse, en France, puis aux États-Unis où il fait la rencontre avec le chorégraphe Paul Sanasardo. Au fil des rencontres qui ont marqué son parcours, Dominique Petit rend compte d'une histoire de la danse méconnue et témoigne d'une réalité de danseur-interprète aux États-Unis dans les années soixante-dix.

This first part of interview, «Years 1970 and 1971: from the edges of the Rhône to New York, first choreographic shocks», looks back on the meeting between Dominique Petit and dance in France, then in the United States where he meets the choreographer Paul Sanasardo. Throughout the encounters that marked his career, Dominique Petit delivers an unknown dance history and speaks also about the reality of being a performer in the United States in the seventies.

\section{INDEX}

Mots-clés : Dominique Petit, Paul Sanasardo, États-Unis, danse

Keywords : Dominique Petit, Paul Sanasardo, United-States, dance 


\section{AUTEURS}

\section{DOMINIQUE PETIT}

Dominique Petit se forme à la modern dance américaine lors d'un séjour de quatre ans à New York auprès de Paul Sanasardo. Il est interprète de sa compagnie pendant trois ans. De retour en France, il rencontre Carolyn Carlson et intègre le Groupe de Recherche Théâtrale de l'Opéra de Paris, dont il restera membre jusqu'au départ de Carolyn Carlson en 1980.

Tout en dansant pour d'autres chorégraphes, (Caroline Marcadé, François Verret, Régine Chopinot) il commence peu à peu son propre travail de création. Il est remarqué au festival d'Avignon en 1981, avec son solo Igor Urstark accompagné par Barre Phillips. Suite à cela, il entreprend plusieurs collaborations avec des musiciens (Barre Phillips, Steve Lacy, Denis Levaillant, John Surman, Hervé Bourde...).

En 1983, la Maison de la Danse de Lyon accueille ses premières créations de groupe : Les pas perdus et Aranzaquil, qui signent le début de sa compagnie. En compagnie d'Anne Carrié il reçoit, en 1985, le 1er Prix du Forum Régional d'île-de-France pour leur duo Jade.

À partir de cette date, la compagnie Dominique Petit reçoit le soutien du Ministère de la Culture au titre de l'aide à la création puis de l'aide aux compagnies. Elle est accueillie dans de nombreux festivals en France (Danse en Yvelines, Festival d'Aix en Provence, Danse Emoi, Les Hivernales d'Avignon...) et à l'étranger (Utrecht, Nüremberg,...).

Il est invité comme professeur et coordinateur des études au CNDC d'Angers de 1985 à 1988. Il enchaîne pendant dix ans les créations et les tournées avec sa compagnie : Les Leçons de L'aube, Frontières... et surtout Les Tournesols, pièce chorégraphique pour six danseurs hommes. En 1996, il est à nouveau invité au CNDC L’Esquisse comme coordinateur des études et décide alors de se consacrer prioritairement à l'enseignement. Titulaire du CA de danse contemporaine, il a enseigné dans de nombreux pays (USA, Chili, Colombie, Israël, Allemagne, Belgique...) et au conservatoire de La Roche-sur-Yon, en poursuivant parallèlement des projets artistiques divers, comme danseur, performer, et chorégraphe : Danser hors de soi de Daniel Dobbels, Objets Trouvés, Sweet suite, East side, Variations sur le Faune, Panique..., Sale défaite, Monsieur Fugue, Philipina.

En 2019, il est engagé par Akram Khan pour sa dernière pièce, Outwitting the Devil, créée au Festival d'Avignon et en tournée internationale pour plusieurs années.

\section{MAËVA LAMOLIÈRE}

Maëva Lamolière a été formée à la danse contemporaine au conservatoire de la Roche sur Yon puis au Trinity Laban Conservatoire de Londres.

Depuis 2015 elle développe ses propres projets chorégraphiques et est interprète pour MarieGabrielle Rotie, Marguerite Danguy des Déserts (DDD compagnie) et collabore régulièrement avec Alain Michard.

Elle obtient son Diplôme d'État en 2017 et enseigne la danse contemporaine (conservatoire de Gennevilliers) et la culture chorégraphique (conservatoire de Gennevilliers et conservatoire de Lille). Depuis septembre 2019, elle accompagne également les élèves de master du CNSMDP de Paris et intervient au sein de la formation DE (Pont Supérieur de Nantes). Après un master 2 au département danse de Paris 8, Maëva commence en octobre 2018 une thèse de doctorat codirigée par Isabelle Launay et Sylviane Pagès et actuellement intitulée : Carlotta Ikeda : Poétique des métamorphoses pour une déconstruction des catégories de genre. Entre la France et le Japon, retour sur une généalogie butô minorée. 